\title{
Lnc-IL7R promotes the growth of fibroblast-like synoviocytes through interaction with enhancer of zeste homolog 2 in rheumatoid arthritis
}

\author{
ZHAO YE ${ }^{1}$, JUAN XU $^{2}$, SHUKUI LI $^{1}$, CHENG CAI $^{1}$, TIEJUN $^{3}{ }^{3}$ and LISHAN SUN ${ }^{1}$ \\ Departments of ${ }^{1}$ Orthopedics, ${ }^{2}$ Ultrasound and ${ }^{3}$ Teaching, Cangzhou Central Hospital, Cangzhou, Hebei 061000, P.R. China
}

Received November 19, 2015; Accepted November 25, 2016

DOI: $10.3892 / \mathrm{mmr} .2017 .6150$

\begin{abstract}
Rheumatoid arthritis (RA) is an inflammatory and autoimmune disease that affects $\sim 1 \%$ of the world's population. Although the precise mechanism of RA has yet to be elucidated, accumulating evidence suggests that fibroblast-like synoviocytes (FLSs) serve critical roles in the initiation and progression of RA. However, the underlying molecular mechanisms of FLS proliferation have yet to be elucidated. Long noncoding-interleukin-7 receptor (lnc-IL7R) has been recently identified, which is activated by lipopolysaccharide (LPS) stimulation and diminishes the LPS-induced inflammatory response. In the present study, gain- and loss-of-function assays were performed in order to investigate the role of lnc-IL7R in FLS. It is demonstrated, to the best of the authors' knowledge for the first time, that Inc-IL7R promotes cell proliferation, cell cycle progression and inhibits apoptosis in FLS. Further investigation identified that lnc-IL7 interacts with enhancer of zeste homolog 2 (EZH2) and is required for polycomb repressive complex 2 (PRC2)-mediated suppression, including cyclin-dependent kinase inhibitor $1 \mathrm{~A}$ and cyclin-dependent kinase inhibitor 2A. Lnc-IL7R may be a promising therapeutic target for the treatment of RA.
\end{abstract}

\section{Introduction}

Rheumatoid arthritis (RA) is an inflammatory and autoimmune disease that affects $\sim 1 \%$ of the world's population. RA is characterized by chronic and invasive synovitis, damage to cartilage and bone, and cartilage destruction $(1,2)$. Despite the advances in RA treatment, including aggressive immune suppression with biologics and modifying anti-rheumatic drugs, the general efficacy of treatment remains unsatisfactory (3). Accumulating evidence suggests that fibroblast-like synoviocytes (FLSs) serve

Correspondence to: Dr Lishan Sun, Department of Orthopedics, Cangzhou Central Hospital, 16 West Xinhua Road, Cangzhou, Hebei 061000, P.R. China

E-mail: sunlishanmyc@126.com

Key words: fibroblast-like synoviocytes, enhancer of zeste homolog 2, rheumatoid arthritis, long noncoding-interleukin-7 receptor, long noncoding RNA critical roles in the initiation and progression of RA (4). The abnormal proliferation of fibroblast-like synoviocytes (FLSs) leads to destructive invasion of the cartilage and bones through secreting digestive proteases and inflammatory cytokines $(5,6)$. Furthermore, FLSs exhibit resistance to apoptosis induced by several apoptotic stimuli $(7,8)$. Therefore, understanding the molecular mechanism of FLS proliferation and developing pro-apoptosis drugs for FLS is important.

The human transcriptome contains a large number of protein-coding mRNAs and noncoding transcripts (9). Among these, long noncoding RNA (lncRNA) is defined as a molecule of RNA >200 nucleotides in length (10). Accumulating studies have reported that numerous lncRNAs have important biological functions, including chromatin remodeling, transcriptional control and posttranscriptional modification $(11,12)$. Dysregulation of lncRNA has been identified to be associated with human diseases, including inflammation and cancer (13-15). The abnormal expression of lncRNA may contribute to disease initiation and progression through regulating expression of the gene products $(16,17)$. A recent study has reported that differentiation and activation of immune cells are associated with lncRNAs, which have an essential role in autoimmune diseases, including RA (18). However, the role of lncRNA in FLSs has yet to be elucidated.

Long noncoding-interleukin-7 receptor (lnc-IL7R) has been recently identified, and it overlaps with the 3 ' untranslated region (3'UTR) of the interleukin-7 receptor $\alpha$-subunit gene (IL7R). Lnc-IL7R is activated by LPS stimulation, and reduces the LPS-induced inflammatory response (19). A previous study (20) demonstrated the differential expression of lncRNAs between RA patients and non-RA patients. From the different expression profiles, it was identified that lnc-IL7R is one of these lncRNAs. However, the role of lnc-IL7R in FLSs has yet to be elucidated. The present study demonstrates that lnc-IL7R promotes cell proliferation, cell cycle progression and inhibits apoptosis in FLSs. Further investigation identified that lnc-IL7 interacts with enhancer of zeste homolog 2 (EZH2), and is required for polycomb repressive complex 2 (PRC2)-mediated suppression.

\section{Materials and methods}

Isolation and culture of FLSs. Isolation and culture of FLSs was performed as previously described (21). In brief, synovial 
tissues were obtained during surgery from RA patients diagnosed according to American Rheumatism Association criteria. The present study was approved by the ethics committee of Cangzhou Central Hospital (Cangzhou, China) and written informed consent was provided by all patients. The synovial tissues were digested with $2.5 \mathrm{~g} / \mathrm{l}$ trypsin for $2 \mathrm{~h}$, and cells were centrifuged at $2,500 \times \mathrm{g}$ for $10 \mathrm{~min}$ at $4^{\circ} \mathrm{C}$ and cultured in Dulbecco's minimum essential medium in $5 \% \mathrm{CO}_{2}$ at $37^{\circ} \mathrm{C}$. Cells within the 3 rd-8th passages were used for all experiments.

MTT assays. A total of $1 \times 10^{3}$ cells per well were seeded in 96-well plates and the viability of the cells was ascertained from three replicates in three independent experiments using an MTT assay [Vazyme Biotech (Nanjing) Co., Ltd., Nanjing, Jiangsu, China] after 3 days.

RNA isolation and reverse transcription-quantitative polymerase chain reaction $(R T-q P C R)$. Total RNA was isolated using Trizol reagent (Invitrogen; Thermo Fisher Scientific, Inc., Waltham, MA, USA) and $1 \mu \mathrm{g}$ complementary DNAS (cDNAs) were synthesized using a Prime Script RT Reagent kit (Takara Bio, Inc., Otsu, Japan) according to the manufacturer's protocol. Quantification of gene expression was determined using FastStart Universal SYBR Green Master mix in the StepOne Real-Time PCR system (Applied Biosystems; Thermo Fisher Scientific, Inc.). The relative expression of RNAs was calculated using the comparative $\mathrm{Cq}$ method (or the $2^{-\Delta \Delta \mathrm{Cq}}$ method) (22). Gene-specific primers were as follows: lnc-IL7R: 5'-CCAGCCTTTGCCTCTTCC TTCAAT-3' (forward), 5'-CCGTACCAAGTCTCTTAGCCC CTC-3' (reverse); p16: 5'-GCCCAACGCACCGAATAG TTA-3' (forward), 5'-ACGGGTCGGGTGAGAGT-3' (reverse); p21: 5'-CCTCATCCCGTGTTCTCCTTT-3' (forward), 5'-GTA CCACCCAGCGGACAAGT-3' (reverse).

Transfection. The cDNA encoding lnc-IL7R was subcloned into pcDNA3.1 vector (Invitrogen; Thermo Fisher Scientific, Inc.). Cells were transfected with the plasmids pcDNA3.1 or pcDNA-lnc-IL7R using Lipofectamine 3000 (Invitrogen; Thermo Fisher Scientific, Inc.) according to the manufacturer's protocol. Cells were transfected and grown for $48 \mathrm{~h}$ prior to the subsequent assays. The small interfering RNA (siRNA) against lnc-IL7R was purchased from Guangzhou RiboBio Co., Ltd. (Guangzhou, China). The target sequence was as follows: AAG AGATATATTTCATCGAGA. Cells were transfected with 10 $0 \mathrm{nM}$ siRNA for $48 \mathrm{~h}$ prior to subsequent assays.

Cell-cycle analysis. Cell-cycle analysis was performed as previously described (23). In brief, cells were permeabilized with $75 \%$ ethanol, and DNA was stained with $50 \mu \mathrm{g} / \mathrm{ml}$ propidium iodide. Cellular DNA content was measured using flow cytometric analysis using a FACScan flow cytometer, and the data were analyzed with Kaluza software (version 1.20; Beckman Coulter, Inc., Brea, CA, USA).

Apoptosis analysis. Cells were trypsinized, resuspended and stained with fluorescein isothiocyanate-conjugated annexin $\mathrm{V}$ and 7-aminoactinomycin D (7-AAD; Nanjing KeyGen Biotech Co., Ltd., Nanjing, China) according to the manufacturer's protocol. Cells were then measured by flow cytometric analysis using the FACScan flow cytometer with the data analyzed with Kaluza software.

Western blot analysis. Cells were lysed in RIPA lysis buffer with cocktail protease inhibitor (Selleck Chemicals, Houston, TX, USA). Samples were separated by SDS-PAGE and transferred onto polyvinylidene fluoride (PVDF) membranes (Merck Millipore, Darmstadt, Germany). The PVDF membranes were blocked with 5\% non-fat milk for $1 \mathrm{~h}$ at room temperature and incubated with primary antibodies $(1: 1,000)$ raised against CDK4 (cat. no. 12790), CDK6 (cat. no. 13331), caspase 3 (cat. no. 9661), poly (ADP-ribose) polymerase (PARP; cat. no. 5625) or GAPDH (cat. no. 2118) overnight at $4^{\circ} \mathrm{C}$, followed by incubation with anti-rabbit secondary antibodies $(1: 10,000$; cat. no. 7074) coupled to horseradish peroxidase (all antibodies purchased from Cell Signaling Technology, Danvers, MA, USA) for $1 \mathrm{~h}$ at room temperature. Signals were visualized with an ECL chemiluminescence system (Pierce ECL Western Blotting Substrate; Thermo Fisher Scientific, Inc.).

RNA immunoprecipitation (RIP) and chromatin immunoprecipitation (ChIP) assays. RIP and ChIP assays were performed using an EZ-Magna RIP RNA-binding protein immunoprecipitation kit and an EZ-Magna ChIP kit, according to the manufacturer's protocol (Merck Millipore). RIP and ChIP products were analyzed by RT-qPCR. ChIP-enriched promoter of target genes was quantified by RT-qPCR using the following primers: p16: Forward: 5'-CGCTAAGTGCTCGGA GTTAATA-3', reverse: 5'-CGACCCTGTCCCTCAAATC-3'; p21: Forward: 5'-GTGTCCAGCGCACCAAC-3', reverse: 5'-TGAGCCTGGCCGAGTTC-3'.

RNA pull-down assays. RNA pull-down assays were performed as described previously (24). Briefly, RNAs were biotin-labeled with the Biotin RNA Labeling mix (Roche Diagnostics, Basel, Switzerland) and transcribed in vitro using T7 RNA polymerase (Roche Diagnostics, Switzerland). Biotinylated RNAs were mixed and incubated with cell lysates for $2 \mathrm{~h}$ at $4^{\circ} \mathrm{C}$. Streptavidin-agarose beads (Thermo Fisher Scientific, Inc., Waltham, MA, USA) were added to each binding reaction, and incubated for $1 \mathrm{~h}$ at room temperature. The beads were then boiled in sodium dodecyl sulfate (SDS) buffer. The eluted proteins were detected by western blot analysis.

Statistical analysis. All statistical analyses were performed by using SPSS 19.0 software (IBM SPSS, Armonk, NY, USA). Student's $t$-test was performed for the results from all assays. In all cases, $\mathrm{P}<0.05$ was considered to indicate a statistically significant difference.

\section{Results}

Overexpression of $\operatorname{lnc}-I L 7 R$ promotes FLS proliferation, induces cell cycle progression and decreases cell apoptosis. To determine the role of 1nc-IL7R in FLSs, gain-of-function studies were performed. The cell proliferation of FLSs with empty vector or lnc-IL7R overexpression was detected by performing an MTT assay. It was identified that lnc-IL7R 

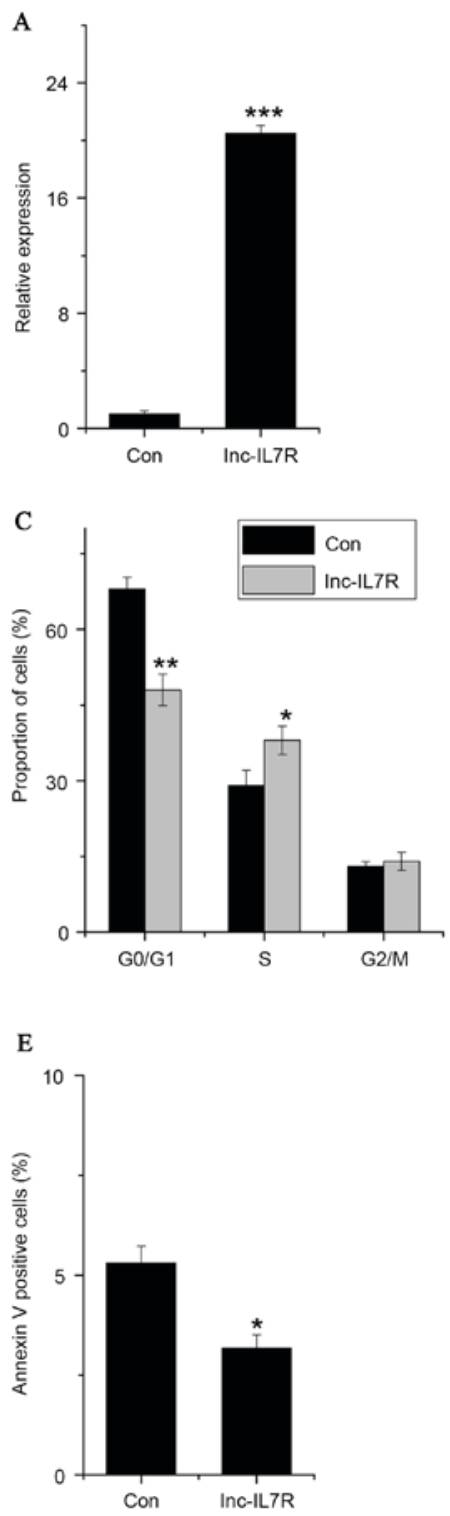

Figure 1. Overexpression of 1nc-IL7R promotes FLS proliferation, induces cell cycle progression and decreases cell apoptosis. (A) Relative expression of lnc-IL7R in FLSs expressing control and lnc-IL7R. (B) Cell growth determined by MTT proliferation assays. (C) Data derived from the FACS analysis, illustrating increases or decreases of the cells in S or G1 phases, respectively, in FLSs with lnc-IL7R overexpression. (D) CDK4 and CDK6 expression levels detected by western blot analysis following 1nc-IL7R overexpression. (E) Cells with 1nc-IL7R overexpression stained with a combination of annexin $\mathrm{V}$ and 7-AAD and analyzed using FACS; cells positive for annexin $\mathrm{V}$ staining were counted as apoptotic cells. (F) Caspase 3 and PARP expression levels were detected by western blot analysis following lnc-IL7R overexpression. Data are shown as the mean \pm standard deviation ( $\mathrm{n}=3$ ). ${ }^{*} \mathrm{P}<0.05,{ }^{* *} \mathrm{P}<0.01,{ }^{* * * *} \mathrm{P}<0.001$ (Student's $t$-test). Lnc-IL7R, long noncoding-interleukin-7 receptor; FLSs, fibroblast-like synoviocytes; MTT, 3-(4,5-dimethylthiazol-2-yl)-2,5-diphenyltetrazolium bromide; FACS, fluorescence-activated cell sorting; CDK4, cyclin-dependent kinase 4; CDK6, cyclin-dependent kinase 6; 7-AAD, 7-amino-actinomycin D; PARP, poly(ADP) ribose polymerase; Con, control.

overexpression increased the proliferation of FLSs compared with control cells (Fig. 1A and B). To understand the mechanism by which lnc-IL7R promotes cell proliferation, the apoptosis and cell cycle distribution of lnc-IL7R-overexpressed FLSs was detected by fluorescence-activated cell sorting (FACS). It was identified that lnc-IL7R overexpression
A

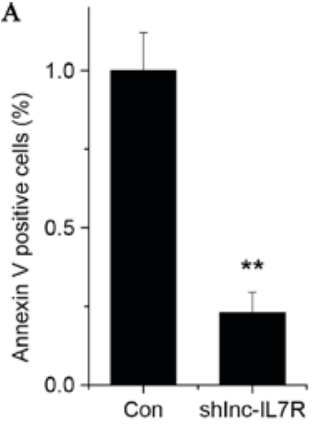

C
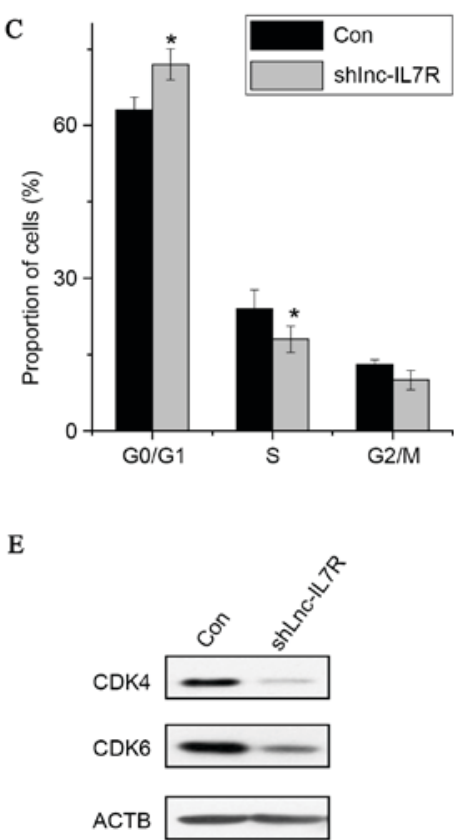

B

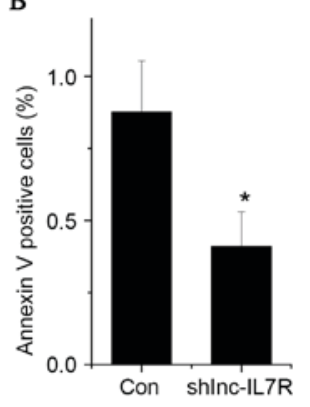

D

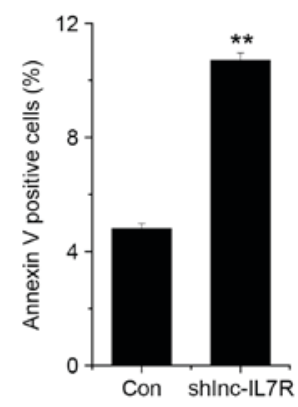

F

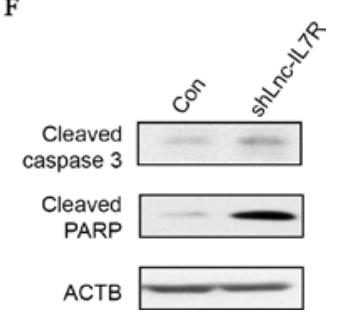

Figure 2. Knockdown of lnc-IL7R inhibits FLS proliferation, induces cell cycle arrest and promotes cell apoptosis. (A) Relative expression of lnc-IL7R in FLSs expressing Con and lnc-IL7R shRNA. (B) Cell growth determined by MTT proliferation assay. (C) FACS analysis showed a marked decrease or increase of cells in the S or G1 phase, respectively, in FLSs with lnc-IL7R shRNA. (D) Cells with lnc-IL7R shRNA stained with a combination of annexin $\mathrm{V}$ and 7-AAD and analyzed by FACS. Cells positive for annexin $\mathrm{V}$ staining were counted as apoptotic cells. (E) CDK4 and CDK6 expression levels detected by western blot analysis following lnc-IL7R silencing. (F) Caspase 3 and PARP expression levels detected by western blot analysis following lnc-IL7R silencing. Data are shown as the mean \pm standard deviation $(\mathrm{n}=3)$. ${ }^{*} \mathrm{P}<0.05,{ }^{* *} \mathrm{P}<0.01$ (Student's $t$-test). Lnc-IL7R, long noncoding-interleukin-7 receptor; FLS, fibroblast-like synoviocyte; shRNA, small hairpin RNA; MTT, 3-(4,5-dimethylthiazol-2-yl)-2,5-diphenyltetrazolium bromide; Con, control; FACS, fluorescence-activated cell sorting; CDK4, cyclin-dependent kinase 4; CDK6, cyclin-dependent kinase 6; 7-AAD, 7-amino-actinomycin D; PARP, poly(ADP) ribose polymerase.

significantly promoted G1/S transition in FLSs (Fig. 1C). Consistent with the FACS data, the expression of G1/S-phase checkpoint markers, including CDK4 and CDK6, were upregulated in lnc-IL7R-overexpressed FLSs (Fig. 1D). However, lnc-IL7R-overexpressed FLSs showed a significantly higher percentage of annexin $\mathrm{V}$-positive cells than did control cells (Fig. 1E). Consistent with the FACS results, apoptosis markers, including cleaved caspase 3 and PARP, markedly decreased in FLSs with lnc-IL7R overexpression (Fig. 1F). Taken together, the results demonstrated that lnc-IL7R promotes FLS proliferation, via driving cell cycle progression and decreasing cell apoptosis. 
A

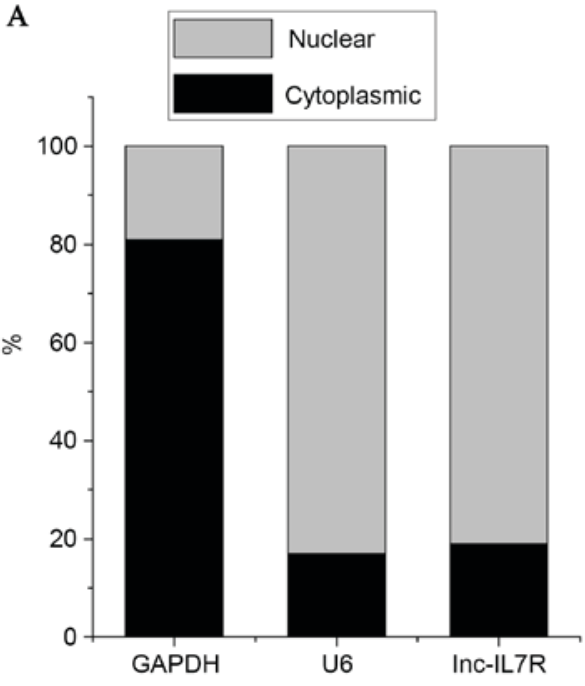

$\mathrm{C}$

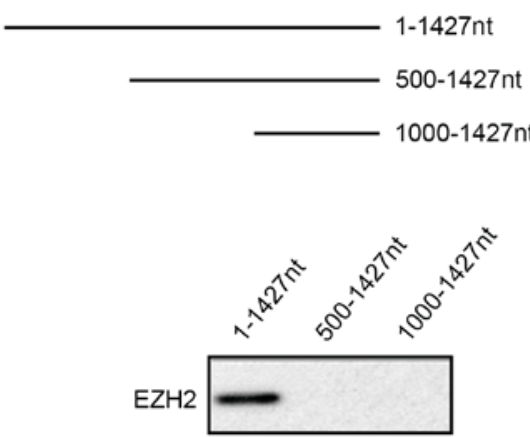

B

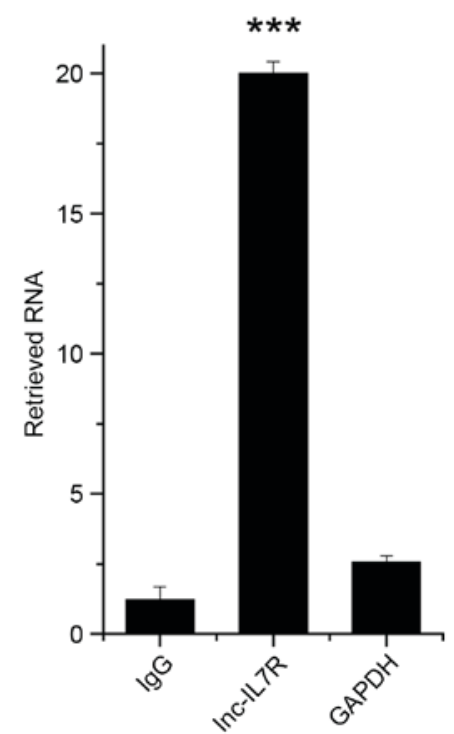

Figure 3. Lnc-IL7R interacts with EZH2. (A) Representative analysis of lnc-IL7R distribution by cellular fractionation of FLSs. GAPDH mRNA and U6 RNA served as controls for cytoplasmic and nuclear RNAs, respectively. (B) Results from RIP and subsequent RT-qPCR assays. Relative quantification of lnc-IL7R and GAPDH in RNA-protein complexes immunoprecipitated with IgG or EZH2 antibodies from nuclear extracts. (C) Deletion mapping of EZH2-binding domain in lnc-IL7R. Western blot of EZH2 in protein samples pulled down by different lnc-IL7R fragments. Data are shown as the mean \pm standard deviation $(\mathrm{n}=3) .{ }^{* * *} \mathrm{P}<0.001$ (Student's $t$-test). Lnc-IL7R, long noncoding-interleukin-7 receptor; EZH2, enhancer of zeste homolog 2; FLSs, fibroblast-like synoviocytes; GAPDH, glyceraldehyde 3-phosphate dehydrogenase; RIP, RNA immunoprecipitation; RT-qPCR, reverse transcription-quantitative polymerase chain reaction; IgG, immunoglobulin G.

Knockdown of Inc-IL7R inhibits FLS proliferation, induces cell cycle arrest and promotes cell apoptosis. Lnc-IL7R expression in FLSs was silenced (Fig. 2A) and an MTT assay was performed to detect the cell proliferation of FLSs with lnc-IL7R small hairpin RNA (shRNA). It was identified that knockdown of lnc-IL7R decreased the proliferative capacity of FLSs compared with control cells (Fig. 2B). Similarly, a FACS assay demonstrated that lnc-IL7R knockdown significantly inhibited G1/S transition and promoted cell apoptosis (Fig. 2C and D). Consistent with the FACS data, the expression of G1/S phase checkpoint markers were markedly downregulated, while the apoptosis markers were upregulated in lnc-IL7R knockdown FLSs (Fig. 2E and F). Collectively, these data indicate that lnc-IL7R serves an important role in FLS proliferation, cell cycle and apoptosis.

lnc-IL7R interacts with EZH2. The molecular mechanisms by which lnc-IL7R promotes cell proliferation were subsequently investigated. First, cellular fractionation assays were performed to analyze the subcellular localization of lnc-IL7R. It was identified that lnc-IL7R was localized in the nuclei of FLSs (Fig. 3A). This suggested that lnc-IL7R may be involved in transcriptional regulation.
A previous study (25) reported that $20 \%$ of human lncRNs are associated with PRC2. It was hypothesized that lnc-IL7R may interact with $\mathrm{EZH} 2$, a core component of PRC2, and regulate gene expression. To confirm this, an RIP assay was performed with an EZH2 antibody from nuclear extracts of FLSs. A marked enrichment of 1nc-IL7R by the EZH2 antibody was identified, but not any enrichment of GAPDH compared with the IgG control antibody (Fig. 3B). Consistent with the RIP assay, the association between EZH2 and 1nc-IL7R was validated through a RNA pull-down assay (Fig. 3C). The deletion-mapping assay showed that 500 nucleotides at the 5 ' end of lnc-IL7R are required for its interaction with EZH2. Collectively, the results demonstrated that there is an interaction between lnc-IL7R and EZH2.

Inc-IL7R promotes FLS proliferation, induces cell cycle progression and decreases cell apoptosis via association with EZH2. To investigate the practical relevance of the interaction of 1nc-IL7R and EZH2, EZH2 was silenced in lnc-IL7R-overexpressed FLSs (Fig. 4A). EZH2 knockdown reversed the effects of promotion of cell proliferation and cell cycle progression, and the anti-apoptosis effects, induced 
A

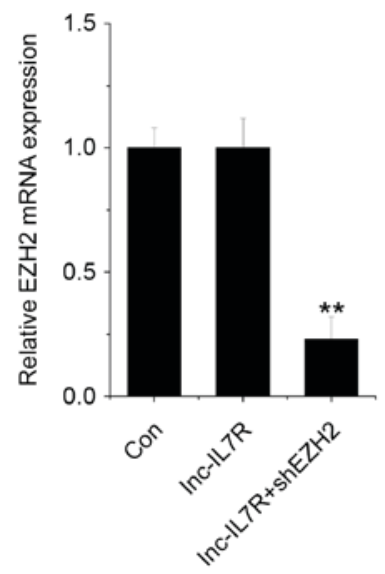

$\mathrm{C}$

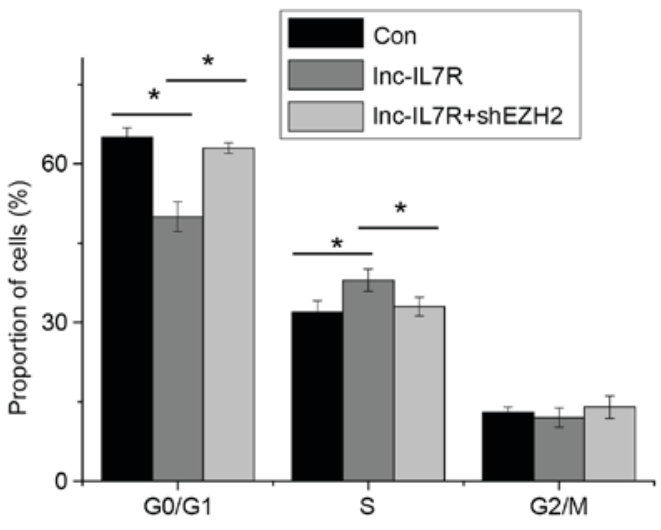

B

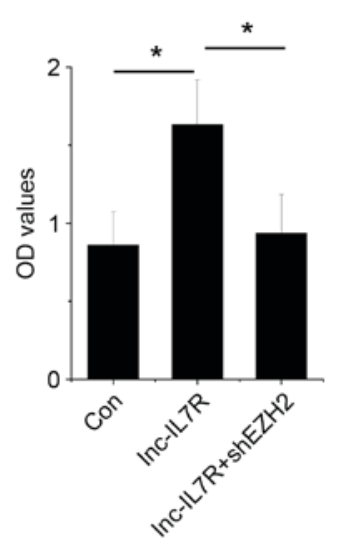

D

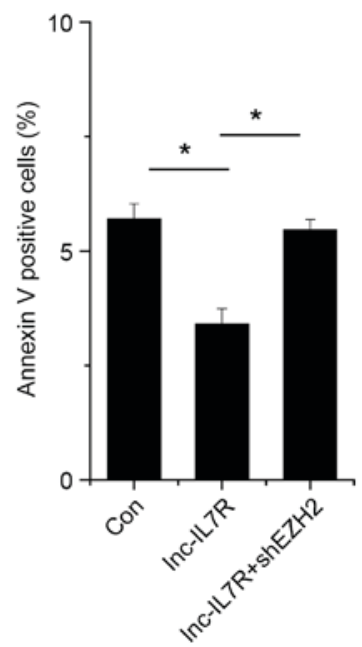

Figure 4. Lnc-IL7R promotes FLS proliferation, induces cell cycle progression and decreases cell apoptosis via association with EZH2. (A) Relative expression of EZH2. (B) Cell growth of indicated groups determined by MTT proliferation assays. (C) Cell cycle distribution detected by FACS analysis. (D) Cell apoptosis detected by FACS analysis. Data are shown as the mean \pm standard deviation ( $\mathrm{n}=3$ ). ${ }^{*} \mathrm{P}<0.05$ (Student's $t$-test). Lnc-IL7R, long noncoding-interleukin-7 receptor; FLSs, fibroblast-like synoviocytes; EZH2, enhancer of zeste homolog 2; MTT, 3-(4,5-dimethylthiazol-2-yl)-2,5-diphenyltetrazolium bromide; FACS, fluorescence-activated cell sorting; Con, control.

by lnc-IL7R (Fig. 4B-D). These data suggest that lnc-IL7R exerts its function, at least in part, through interaction with EZH2.

Lnc-IL7R regulate target gene expression via epigenetic mechanisms. To further corroborate the practical relevance of the association between lnc-IL7R and EZH2, PRC2 target genes, which regulate the cell cycle, were detected, including cyclin dependent kinase inhibitor 2A (p16) and cyclin dependent kinase inhibitor 1A (p21) (Fig. 5A). p16 and p21 expression levels were decreased by lnc-IL7R overexpression, and reversed by knockdown of EZH2. By contrast, both p16 and p21 expression levels were increased by lnc-IL7R knockdown (Fig. 5B). A ChIP assay in lnc-IL7R-overexpressed FLSs was performed to test whether lnc-IL7R is involved in transcriptional regulation by recruiting EZH2 to target genes. The results demonstrated that lnc-IL7R increased the binding of EZH2 and H3K27me 3 levels across the p16 and p21 promoter regions (Fig. 5C and D).

\section{Discussion}

Development of RA is closely associated with characteristic changes of the synovium, including FLS hyperplasia, abnormal angiogenesis and infiltration of immune cells $(26,27)$. Previous studies $(28,29)$ have reported that the excessive proliferation of FLSs is associated with aberrant activation of the inflammatory signal pathway. Noncoding RNA (ncRNA) is also involved in regulating FLS proliferation. For example, microRNA (miR)-152 overexpression significantly decreases FLS proliferation through the upregulation of SFRP4, a negative regulator of the Wnt signaling pathway (30). However, the function of lncRNA in FLSs has yet to be elucidated. The present study demonstrated that lnc-IL7R promotes cell proliferation and cell cycle progression, and inhibits apoptosis in FLSs. Although the present study has demonstrated that lnc-IL7R diminishes the LPS-induced inflammatory response in human monocytes, this phenomenon was not observed in FLSs (data not shown). 

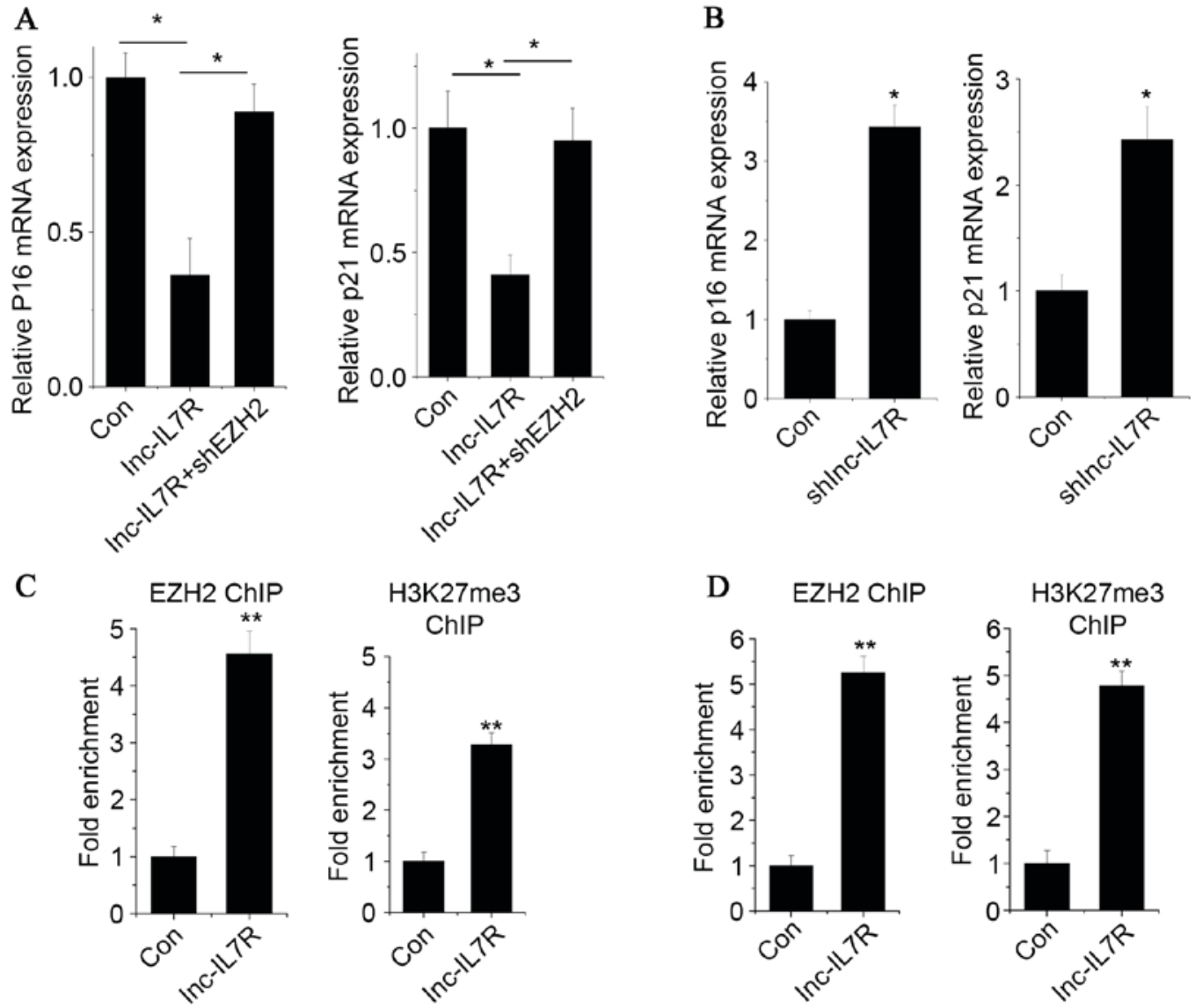

Figure 5. Lnc-IL7R regulate target gene expression via epigenetic mechanisms. The (A) p16 and (B) p21 expression levels of the groups as detected by RT-qPCR. (C) The occupancy of EZH2 and H3K27me3 in the promoter regions of p16 measured by EZH2 and H3K27me3 ChIP assay followed by RT-qPCR. (D) The occupancy of EZH2 and H3K27me3 in the promoter regions of p21 was measured by EZH2 and H3K27me3 ChIP assay followed by RT-qPCR. Data are shown as mean \pm standard deviation; $\mathrm{n}=3$. ${ }^{*} \mathrm{P}<0.05,{ }^{* *} \mathrm{P}<0.01,{ }^{* * *} \mathrm{P}<0.001$ (Student's $t$-test). Lnc-IL7R, long noncoding-interleukin-7 receptor; p16, cyclin-dependent kinase inhibitor 2A; p21, cyclin-dependent kinase inhibitor 1A; RT-qPCR, reverse transcription-quantitative polymerase chain reaction; EZH2, enhancer of zeste homolog 2; H3K27me3, histone 3 lysine 27 trimethylation; ChIP, chromatin immunoprecipitation; Con, control.

Lnc-IL7R may therefore exert its function in a tissue-specific manner.

Aberrant epigenetic modifications have been thoroughly elucidated in human diseases. Epigenetic modifications, including DNA methylation and histone modification, regulate gene expression $(31,32)$. Previous studies $(33,34)$ have shown an extensive aberrant methylation of gene promoters in FLSs, including $\mathrm{C}-\mathrm{X}-\mathrm{C}$ motif chemokine ligand 12, interleukin 10 and interleukin 6. Many of these genes are involved in the immune response and in inflammation $(35,36)$. However, histone modifications involved in RA have yet to be elucidated $(37,38)$. A previous study (39) demonstrated that EZH2 is overexpressed in FLSs, and inhibits the expression of secreted frizzled-related protein 1 . However, none of the PRC2 core components are responsible for the sequence-specific DNA-binding activity. Previous studies $(40,41)$ have reported that PRC2 may be recruited to target genes by lncRNA. In the present study, it was demonstrated that lnc-IL7R associates with EZH2. Lnc-IL7R depletion leads to upregulation of genes which are suppressed by EZH2, including p16 and p21. Additionally, lnc-IL7R overexpression increases the binding of EZH2 and H3K27me3 levels across p16 and p21 promoters. Overall, the present study proposes a model in which lncRNA regulates proliferation via association with EZH2. Whether lnc-IL7R is associated with other proteins requires further investigation. Understanding the precise molecular mechanisms by which lnc-IL7R functions in FLSs will aid the exploration of new therapies for RA.

\section{Acknowledgements}

This study was supported by a grant from the Support Project of Hebei province science and technology (grant no. $1123038 \mathrm{ZD})$.

\section{References}

1. Gibofsky A: Epidemiology, pathophysiology, and diagnosis of rheumatoid arthritis: A Synopsis. Am J Manag Care 20: S128-S135, 2014.

2. Huber LC, Distler O, Tarner I, Gay RE, Gay S and Pap T: Synovial fibroblasts: Key players in rheumatoid arthritis. Rheumatology (Oxford) 45: 669-675, 2006.

3. Yang CL, Or TC, Ho MH and Lau AS: Scientific basis of botanical medicine as alternative remedies for rheumatoid arthritis. Clin Rev Allergy Immunol 44: 284-300, 2013.

4. Mor A, Abramson SB and Pillinger MH: The fibroblast-like synovial cell in rheumatoid arthritis: A key player in inflammation and joint destruction. Clin Immunol 115: 118-128, 2005.

5. Bottini N and Firestein GS: Duality of fibroblast-like synoviocytes in RA: Passive responders and imprinted aggressors. Nat Rev Rheumatol 9: 24-33,2013. 
6. Bartok B and Firestein GS: Fibroblast-like synoviocytes: Key effector cells in rheumatoid arthritis. Immunol Rev 233: 233-255, 2010.

7. Smith MD and Walker JG: Apoptosis a relevant therapeutic target in rheumatoid arthritis? Rheumatology (Oxford) 43: 405-407, 2004.

8. Bai S, Liu H, Chen KH, Eksarko P, Perlman H, Moore TL and Pope RM: NF-kappaB-regulated expression of cellular FLIP protects rheumatoid arthritis synovial fibroblasts from tumor necrosis factor alpha-mediated apoptosis. Arthritis Rheum 50: 3844-3855, 2004.

9. Wilusz JE, Sunwoo H and Spector DL: Long noncoding RNAs: Functional surprises from the RNA world. Genes Dev 23 1494-1504, 2009.

10. Liu G, Mattick JS and Taft RJ: A meta-analysis of the genomic and transcriptomic composition of complex life. Cell Cycle 12: 2061-2072, 2013.

11. Huang JL, Zheng L, Hu YW and Wang Q: Characteristics of long non-coding RNA and its relation to hepatocellular carcinoma. Carcinogenesis 35: 507-514, 2014

12. Lam MT, Li W, Rosenfeld MG and Glass CK: Enhancer RNAs and regulated transcriptional programs. Trends Biochem Sci 39: 170-182, 2014.

13. Li Y, Chen H, Pan T, Jiang C, Zhao Z, Wang Z, Zhang J, Xu J and Li X: LncRNA ontology: Inferring lncRNA functions based on chromatin states and expression patterns. Oncotarget 24: 39793-39805, 2015.

14. Keenan CR, Schuliga MJ and Stewart AG: Pro-inflammatory mediators increase levels of the noncoding RNA GAS5 in airway smooth muscle and epithelial cells. Can J Physiol Pharmacol 93: 203-206, 2015.

15. Cheng HS, Njock MS, Khyzha N, Dang LT and Fish JE: Noncoding RNAs regulate NF- $\kappa \mathrm{B}$ signaling to modulate blood vessel inflammation. Front Genet 5: 422, 2014.

16. Jiang H, Modise T, Helm R, Jensen RV and Good DJ: Characterization of the hypothalamic transcriptome in response to food deprivation reveals global changes in long noncoding RNA and cell cycle response genes. Genes Nutr 10: 48, 2015.

17. Xiao H, Tang K, Liu P, Chen K, Hu J, Zeng J, Xiao W, Yu G, Yao W, Zhou H, et al: LncRNA MALAT1 functions as a competing endogenous RNA to regulate ZEB2 expression by sponging miR-200s in clear cell kidney carcinoma. Oncotarget 6 : 38005-38015, 2015.

18. Spurlock CF III, Tossberg JT, Matlock BK, Olsen NJ and Aune TM: Methotrexate inhibits NF- $\kappa B$ activity via long intergenic (noncoding) RNA-p21 induction. Arthritis Rheumatol 66: 2947-2957, 2014

19. Cui H, Xie N, Tan Z, Banerjee S, Thannickal VJ, Abraham E and Liu G: The human long noncoding RNA lnc-IL7R regulates the inflammatory response. Eur J Immunol 44: 2085-2095, 2014

20. Messemaker TC, Frank-Bertoncelj M, Marques RB, Adriaans A, Bakker AM, Daha N, Gay S, Huizinga TW, Toes RE, Mikkers HM and Kurreeman F: A novel long non-coding RNA in the rheumatoid arthritis risk locus TRAF1-C5 influences C5 mRNA levels. Genes Immun 17: 85-92, 2016.

21. Sun J, Yan P, Chen Y, Chen Y, Yang J, Xu G, Mao H and Qiu Y: MicroRNA-26b inhibits cell proliferation and cytokine secretion in human RASF cells via the Wnt/GSK-3 $\beta / \beta$-catenin pathway. Diagn Pathol 10: 72, 2015.

22. Livak KJ and Schmittgen TD: Analysis of relative gene expression data using real-time quantitative PCR and the 2(-Delta Delta C(T)) method. Methods 25: 402-408, 2001.

23. Braconi C, Valeri N, Kogure T, Gasparini P, Huang N, Nuovo GJ, Terracciano L, Croce CM and Patel T: Expression and functional role of a transcribed noncoding RNA with an ultraconserved element in hepatocellular carcinoma. Proc Natl Acad Sci USA 108: 786-791, 2011.

24. Cao C, Sun J, Zhang D, Guo X, Xie L, Li X, Wu D and Liu L: The long intergenic noncoding RNA UFC1, a target of MicroRNA $34 \mathrm{a}$,interacts with the mRNA stabilizing protein $\mathrm{HuR}$ to increase levels of $\beta$-catenin in HCC cells. Gastroenterology 148: 415-426 e18, 2015.
25. Khalil AM, Guttman M, Huarte M, Garber M, Raj A Rivea Morales D, Thomas K, Presser A, Bernstein BE, van Oudenaarden A, et al: Many human large intergenic noncoding RNAs associate with chromatin-modifying complexes and affect gene expression. Proc Natl Acad Sci USA 106 11667-11672, 2009

26. Sweeney SE and Firestein GS: Rheumatoid arthritis: Regulation of synovial inflammation. Int J Biochem Cell Biol 36: 372-378, 2004.

27. Umar S, Hedaya O, Singh AK and Ahmed S: Thymoquinone inhibits TNF- $\alpha$-induced inflammation and cell adhesion in rheumatoid arthritis synovial fibroblasts by ASK1 regulation. Toxicol Appl Pharmacol 287: 299-305, 2015

28. Wang H, Fang Y, Wang Y, Wang Z, Zou Q, Shi Y, Chen J and Peng D: Inhibitory effect of curcumol on Jak2-STAT signal pathway molecules of fibroblast-like synoviocytes in patients with rheumatoid arthritis. Evid Based Complement Alternat Med 2012: 746426, 2012.

29. Carrion M, Juarranz Y, Martínez C, González-Álvaro I, Pablos JL, Gutiérrez-Cañas I and Gomariz RP: IL-22/IL-22R1 axis and S100A8/A9 alarmins in human osteoarthritic and rheumatoid arthritis synovial fibroblasts. Rheumatology (Oxford) 52: 2177-2186, 2013

30. Miao CG, Yang YY, He X, Huang C, Huang Y, Qin D, Du CL and Li J: MicroRNA-152 modulates the canonical Wnt pathway activation by targeting DNA methyltransferase 1 in arthritic rat model. Biochimie 106: 149-156, 2014.

31. Picascia A, Grimaldi V, Pignalosa O, De Pascale MR, Schiano C and Napoli C: Epigenetic control of autoimmune diseases: From bench to bedside. Clin Immunol 157: 1-15, 2015.

32. Gonzalez S, Aguilera S, Urzúa U, Quest AF, Molina C, Alliende C, Hermoso M and González MJ: Mechanotransduction and epigenetic control in autoimmune diseases. Autoimmun Rev 10: 175-179, 2011

33. Karouzakis E, Rengel Y, Jüngel A, Kolling C, Gay RE, Michel BA, Tak PP, Gay S, Neidhart M and Ospelt C: DNA methylation regulates the expression of CXCL12 in rheumatoid arthritis synovial fibroblasts. Genes Immun 12: 643-652, 2011.

34. Lin SY, Hsieh SC, Lin YC, Lee CN, Tsai MH, Lai LC, Chuang EY, Chen PC, Hung CC, Chen LY, et al: A whole genome methylation analysis of systemic lupus erythematosus: Hypomethylation of the IL10 and IL1R2 promoters is associated with disease activity. Genes Immun 13: 214-220, 2012.

35. Wilkinson J: Study reveals a DNA methylome signature in rheumatoid arthritis. Epigenomics 4: 481, 2012.

36. Nakano K, Whitaker JW, Boyle DL, Wang W and Firestein GS: DNA methylome signature in rheumatoid arthritis. Ann Rheum Dis 72: 110-117, 2013

37. Viatte S, Plant D and Raychaudhuri S: Genetics and epigenetics of rheumatoid arthritis. Nat Rev Rheumatol 9: 141-153, 2013.

38. Huber LC, Brock M, Hemmatazad H, Giger OT, Moritz F, Trenkmann M, Distler JH, Gay RE, Kolling C, Moch H, et al: Histone deacetylase/acetylase activity in total synovial tissue derived from rheumatoid arthritis and osteoarthritis patients. Arthritis Rheum 56: 1087-1093, 2007.

39. Trenkmann M, Brock M, Gay RE, Kolling C, Speich R, Michel BA, Gay S and Huber LC: Expression and function of EZH2 in synovial fibroblasts: Epigenetic repression of the Wnt inhibitor SFRP1 in rheumatoid arthritis. Ann Rheum Dis 70: 1482-1488, 2011.

40. Yang F, Zhang L, Huo XS, Yuan JH, Xu D, Yuan SX, Zhu N, Zhou WP, Yang GS, Wang YZ, et al: Long noncoding RNA high expression in hepatocellular carcinoma facilitates tumor growth through enhancer of zeste homolog 2 in humans. Hepatology 54: 1679-1689, 2011

41. Kogo R, Shimamura T, Mimori K, Kawahara K, Imoto S, Sudo T, Tanaka F, Shibata K, Suzuki A, Komune S, et al: Long noncoding RNA HOTAIR regulates polycomb-dependent chromatin modification and is associated with poor prognosis in colorectal cancers. Cancer Res 71: 6320-6326, 2011. 